\title{
Input Devices in Mental Health Applications: Steering Performance in a Virtual Reality Paths with WiiMote
}

\author{
Maja Wrzesien ${ }^{1}$, María José Rupérez ${ }^{1}$, and Mariano Alcañiz ${ }^{1,2}$ \\ ${ }^{1}$ Instituto Interuniversitario de Investigación en Bioingeniería y Tecnología Orientada al Ser \\ Humano, Universidad Politécnica de Valencia \\ Camino de Vera s/n, 46022 Valencia, Spain \\ ${ }^{2}$ CIBER, Fisiopatología Obesidad y Nutrición, CB06/03 Instituto de Salud Carlos III, Spain
}

\begin{abstract}
Recent studies present Virtual Reality (VR) as potentially effective technology in the Mental Health (MH) field. The objective of this paper is to evaluate two interaction techniques (traditional vs novel) using a popular and low-cost input device (WiiMote) within a theoretical framework of the Steering Law. The results show that the WiiMote responds to the requirements for the $\mathrm{MH}$ technologies, and that the law of Steering continues to be valid on all of three paths. This opens up a new range of possible research studies for the design and evaluation of interaction techniques in $\mathrm{MH}$ field.
\end{abstract}

Keywords: Mental health, virtual reality, steering law.

\section{Introduction}

Even though the use of 3D input device is increasing, the development of input device for particular domain such as $\mathrm{MH}$ applications evolves slowly [1]. Indeed, there has been few studies performed regarding VR technology within the medical context [2], nor much information regarding the choices of the optimal for this specific context input/output device has been proposed [3]. Moreover, to the authors' knowledge, no studies regarding input devices in mental health VR applications have been performed.

The purpose of this paper is to choose the input device that might correspond to the requirements of VR mental health technologies and evaluate it in the context of a basic task (i.e., traveling task) used in VR mental health applications. More specifically, our aim is to extend the application of a formal predictive model (Steering Law) to a more complex and more close to a real-use path, a maze path. In our opinion, circular or straight paths do not reflect the paths that are habitually used in VR mental health applications, such as those that might be used for virtual cognitive rehabilitation tasks, virtual reality exposure therapies, or therapeutic virtual games. This has motivated us to study the validity of the Steering Law in a maze path.

Virtual Reality has a great potential in different MH applications, and has been effectively used in differ MH disorders [4] such as anxiety disorders; eating disorders; and sexual disorders. Many potential techniques have been proposed to interact with VR environments [5], from standard techniques such as mouse, joystick, touchscreen, to specialist techniques such as spacemouse, trackballs, wands, gloves. Some general 
requirements regarding the input device design for the VR environments have been proposed [6]. However, the input devices used in mental health VR applications should take into account some additional design recommendations, closely related to general guidelines for $\mathrm{MH}$ technologies [7]. First, the input device should be attractive and engaging. Since engagement is one of the critical factors in creating client-therapist relationship, attractive and fun input device might be a first step in engaging clients in the interaction with technology that in turn might help them getting engage in the treatment and relationship with the therapist. Second, the input device should be intuitive and easy to use. Indeed, since the clients have different social and cultural backgrounds, the input device should be easy to use and to learn. Moreover, according to Doherty et al. [7], it seems to be beneficial for the clients to use familiar for them and easily available technologies, since technology is rarely used in day-to-day client work or in therapist training. Third, the input device should be affordable. In fact, interacting in a VR environment often involves the use of devices intentionally made for that purpose. However, these devices are generally expensive and often not available for the average consumer. Finally, the input device should be flexible, allowing the designers to program it in different ways. Indeed, an important requirement for the $\mathrm{MH}$ technology to be of practical use corresponds to the necessity for the system to be adapted to the brand range of MH disorders [8]. Thus, the flexibility of the input device would allow the designers to adapt it according to the needs of a specific application.

\section{Evaluation}

The Steering Law [9] is a model of human movement that predicts the speed and total time spent by a user who steers with a pointing device through a tunnel or along a path presented on a screen. It describes a linear relationship between the mean time of steering along the path and the index of difficulty of the path, which is the ratio between the length and width of the path. In other words, the longer the path and the smaller the width, the greater the mean steering time. This predictive model was chosen as a framework for this evaluation for the following reasons. First, it brings consistency to empirical evaluations of input devices by using rigorous methodology. Second, it gives researchers the opportunity to compare their results in a cross-study comparison. Finally, it allows to extend its application in a 3D straight and circular paths [10] to a path that is more representative in the MH applications (a maze path).

The WiiMote input device [11] was chosen for this study for the following reasons. First, this type of device is a low-cost and currently used 3D input device. Thus, its use seems interesting in terms of a low financial impact and quite high popularity which corresponds to the affordability, familiarity and attractiveness requirements. Second, the WiiMote presents many different possibilities in terms of interactions techniques (for 2D and 3D tasks). Finally, the WiiMote presents a wide range of possible functionalities to be programmed, which corresponds to the flexibility requirement. More specifically, in the case study presented in the following sections the WiiMote input device was programmed according to Bowman et al. classification [6]: input device with (a) both discrete components (buttons) for direction/target selection task, and continuous components (gesture-based movement, i.e. wrist movements) for velocity/acceleration selection, which we can call a traditional 
interaction technique; (b) continuous components (gesture-based movement, i.e. wrist movements) for both direction/target selection task and velocity/acceleration selection, which can be called a novel interaction technique.

The traveling task in a maze path was chosen as the task used in these empirical study for the following reasons. First, traveling, which is a motor component of navigation [6], supports a cognitive component of the navigational process, as well as other interaction-related and therapy-related processes. Second, traveling is a basic task in many VR mental health applications in order to move from a current location to another target location, and support other type of tasks. Finally, travel in a maze path seems better reflect the characteristics of $\mathrm{MH}$ applications than the previously basic shapes used in this theoretical framework (straight and circular). The travel task in a maze path such as a supermarket environment or a city center can be applied in the $\mathrm{MH}$ applications such as cognitive rehabilitation, preventive systems for dementia, or VR post traumatic stress disorder treatment.

\section{Methodology}

A fully-crossed, within-subject factorial design with repeated measures was used. The independent variables were the following: (a) the path shape with three modalities ( $\mathrm{P}=$ straight, circular and maze); (b) the interaction technique (IT=novel vs traditional); and (c) the index of difficulty defined by different path widths and the same path length, with six modalities (ID=122, 144, 168, 190, and $212 \mathrm{~cm}$ ). The steering time and error rates were the dependant variables. Moreover, users' short post-experience semi-directive interview data were collected. In order to avoid the learning process between trials, the order of use of the input device, index of difficulty, as well as the path shape presentation was balanced according to a Latin square pattern. Also, the entrance sides to the maze path and the turning direction for the circular path were changed between the two input devices. Touching the walls of the paths resulted in an error being recorded. According to Zhai and Woltjer [10], only the trials without errors were taken into account in the data analysis. The error rates were analyzed separately.

Eighteen people (seven men and eleven women; 19-36 years old (MD=25,50, $\mathrm{SD}=5,15)$ ) participated in this study. The participants were selected by previously fixed exclusion criteria (left handed people, significant experience in tridimensional and in basic computer games, significant experience in 3D graphics, and significant experience in use of the WiiMote). According to recommendations of Doherty et al. ${ }^{11}$ the evaluation was performed with non-clinical population; however the sample aimed at reflecting the clinical population characteristics (i.e., diversity in social and cultural background; diversity in age; and small experience with innovative technologies).

Three different path shapes were used in the experiment: a straight path, a circular path (see Figure 1 (a) and (b)), and a maze path (see Figure 1 (c)). Six different widths of the path were set for each path shape at 100,122,144,168,190, and $212 \mathrm{~cm}$ simulating paths in which a human could move. The width of the avatar used to navigate in the virtual environment was $45 \mathrm{~cm}$ simulating a human body. The avatar was placed equidistant from the two walls of the paths. The virtual environment for the experiment was developed under 3dGamestudio, version 7.77. 


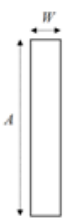

(a)

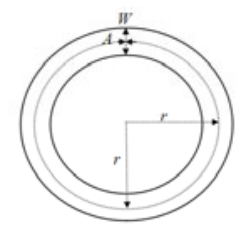

(b)

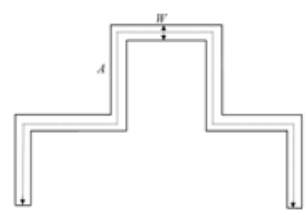

(c)

Fig. 1. The three paths used in the experiment: straight (a), circular (b), and maze (c)

The image of the paths was projected on a $180 \times 240 \mathrm{~cm}$ screen with 1024 by 768 resolution. The output device was chosen as the most often used device for the VR mental health applications in our research center. The task consisted of traveling through a virtual environment toward the clearly marked finishing point. No time pressure or constraints were imposed during the trials; however, the participants were asked to perform the task as fast as possible without touching the walls, restriction needed to validate the Steering Law.

Two different set-ups of the WiiMote were tested: a novel interaction technique involving no use of any buttons (only wrist movements; see Figure 2 (a)), and a traditional interaction technique involving use of buttons (see Figure 2 (b)).

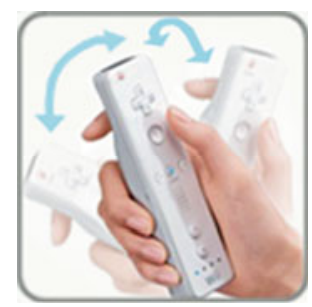

(a)

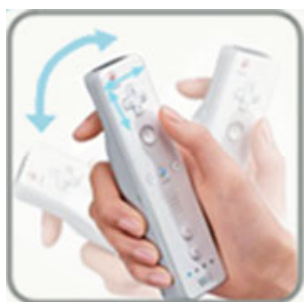

(b)

Fig. 2. Two WiiMote set-ups (novel (a) vs traditional (b)). The bold blue arrows in Figure (a) indicate user motion while navigating and accelerating with the device involving no use of any buttons, only wrist movements, the bold blue arrows in Figure (b) indicate user motion while accelerating, and the thin blue arrows indicate directions of the arrow buttons used for traveling.

\section{Results}

Figure 3 (a, b, c, d, e, f) show the regression results between the index of difficulty and the mean completion time for both interaction techniques and for all paths. The index of difficulty was calculated by dividing the path length by six different path widths. As Figures 3(a) and 3(b) show, there was a strong linear relationship between the mean time and index of difficulty for the straight path with the traditional 
interaction technique $\left(r^{2}=0.996\right)$, and with the novel interaction technique $\left(r^{2}=0.985\right)$. Figures 3 (c) and 3(d) show a slightly different but still strong linear relationship between the mean time and index of difficulty for the circular path $\left(r^{2}=0.915\right.$ for traditional interaction technique, and $r^{2}=0.988$ for the novel interaction technique). Finally, Figures 3(e) and 3(f) show a strong linear relationship between the mean time and the index of difficulty for the maze path with the traditional interaction technique $\left(r^{2}=0.991\right)$ and with the novel interaction technique $\left(r^{2}=0.962\right)$.

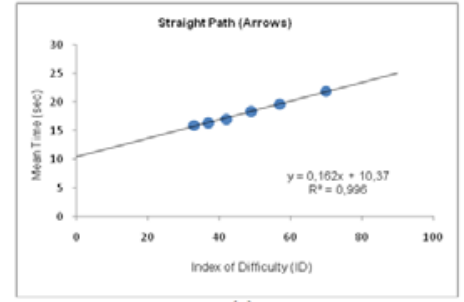

(a)

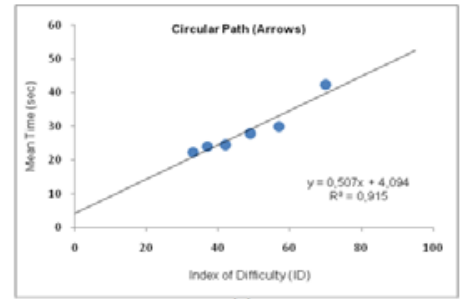

(c)

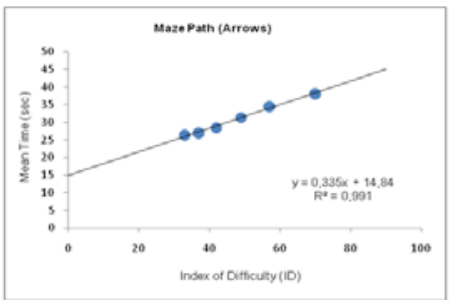

(e)

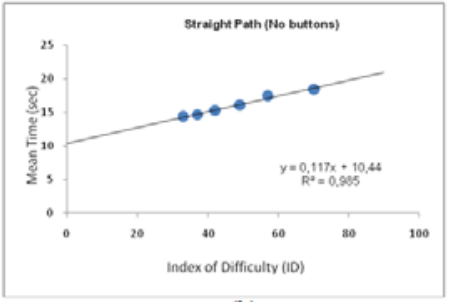

(b)

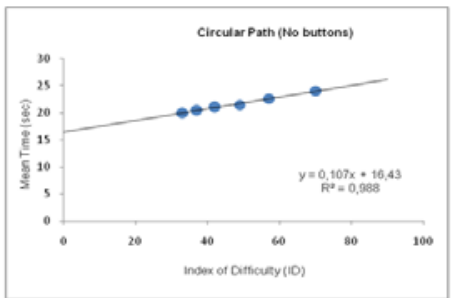

(d)

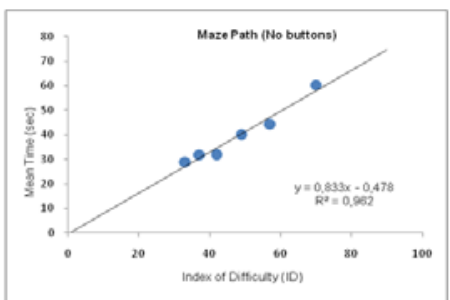

(f)

Fig. 3. Index of Difficulty vs trial completion time in the straight path with traditional (a) and novel interaction technique (b); in the circular path with traditional (c), and novel interaction technique (d); and in the maze path with traditional (e), and novel interaction technique (f)

The highest performance related to the traditional interaction technique in term of slope (less time increase per unit of ID, corresponding to $1 / b$ in steering law equation) was for the straight path ( $\mathrm{IP}=1 / 0.162)$, followed by the maze path $(\mathrm{IP}=1 / 0.335)$, and the lowest performance was for the circular path $(\mathrm{IP}=1 / 0.507)$. The performance related to the novel interaction technique in term of slope was highest for the circular path $(\mathrm{IP}=1 / 0.107)$, followed by the straight path $(\mathrm{IP}=1 / 0.117)$, and then the maze path $(\mathrm{IP}=1 / 0.833)$. 
Due to the differences in the programming set-up of the direction selection (input device set-ups with mixed components, with arrow buttons and wrist movements vs input device set-ups with continuous components, with only wrist movements), the input device set-ups cannot be compared using the slope and the intercept that are derived from the Steering Law equation. However, the two interaction techniques can be compares in terms of error rates. The results show (see Figure 4(a)) that the error rate depends on the path width $\left(\mathrm{F}_{5,85}=53,362, \mathrm{p}<.000\right)$ with significantly more errors for the narrowest path than for the other paths (post hoc multiple comparison with Bonferroni correction, $\mathrm{p}<.0001)$.

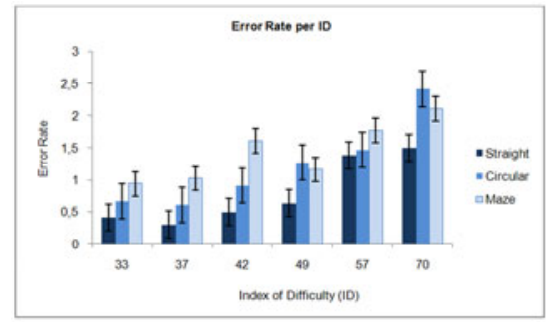

(a)

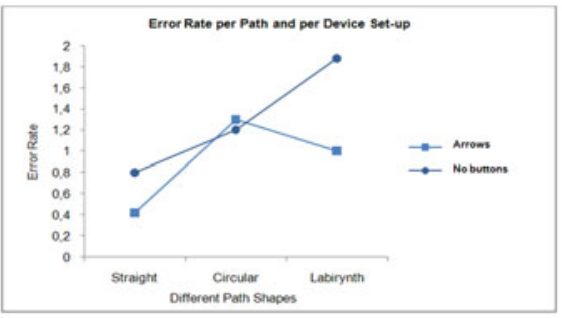

(b)

Fig. 4. The error rates for both interaction techniques regarding different path shapes and different indexes of difficulties (a), and regarding different path shapes

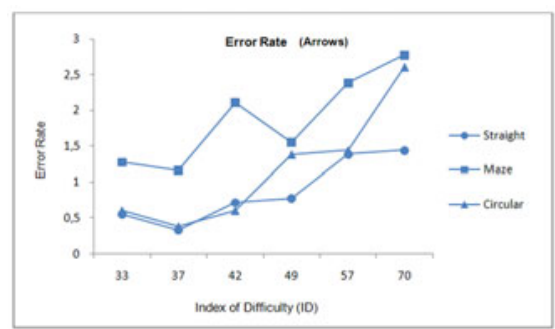

(a)

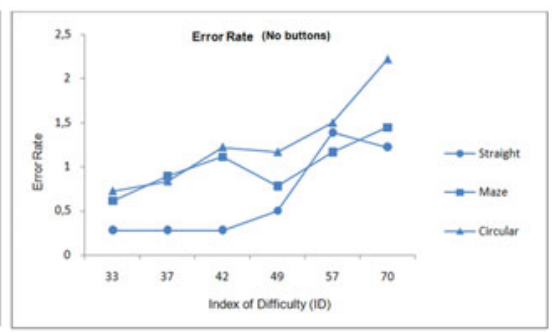

(b)

Fig. 5. The error rates for traditional (a), and for novel interaction technique (b), regarding different path shapes and different indexes of difficulties

In addition, as the results in Figure 4(b) show, the error rate depends on the different interaction technique (novel vs traditional) $\left(\mathrm{F}_{5,85}=5,923, \mathrm{p}<.026\right)$ with significantly more errors for the novel interaction technique (where only wrist was involved). More specifically, the error rates were significantly higher in the maze path than in the other two (post hoc multiple comparison with Bonferroni correction, $\mathrm{p}<.026)$. Finally, there was a significant interaction $\left(\mathrm{F}_{10,170}=3,501, \mathrm{p}<.000\right)$ between the path condition (straight, circular, maze) and the path width in both interaction techniques (see Figure 5(a) and (b)).

The informal interview revealed that the participants preferred the novel interaction technique slightly more than the traditional interaction technique ( $57 \%$ vs $43 \%$ ). The 
majority of participants considered the novel interaction technique fun and intuitive ("I liked this technique because it was new and entertaining"; "It was fun to navigate like this"). Some of the participants that had difficulties in using the novel interaction technique considered that once they used to it, it was not so difficult ("Well, in the beginning it was hard, but once you get used to it it's quite fun"). On the other hand, the participants that chose the traditional interaction technique considered it easy to use and familiar ("I liked it because it was the same thing as playing computer games on the keyboard"; "I preferred this technique because it's like using the remote control"). In general, participants enjoyed traveling with both interaction techniques ("It was so fun to walk in virtual environments"; "Call me for the next study").

\section{Discussion and Conclusions}

The objective of the study was to evaluate an adapted to $\mathrm{MH}$ applications input device in a case study of traveling task. The results show that the WiiMote responds very well to all requirements. First, according to the informal interviews, the traveling task with different WiiMote set-ups was enjoyed and perceived as fun by all participants. Since positive emotional response such as enjoyment is an integral part of engagement [12], we can therefore consider that the WiiMote input-device can engage users. Second, the WiiMote set-ups were intuitive to use and easy to learn. Although, both interaction techniques were appreciate equally by the participants, the results show that there were significantly more errors for novel interaction technique (involving wrist movements) than for traditional interaction technique (involving use of arrow-buttons and wrist movements). On the other hand, the error rates obtained with the traditional interaction technique were significantly greater for the narrowest circular path (see Figure 5a). Therefore, we can conclude that the choice between these two interaction techniques and its usefulness depends on the path shape in a particular application. Finally, WiiMote responds to the flexibility requirement. In this study we evaluate two WiiMote set-ups; however, WiiMote can be programmed in many ways. For instance, a different interaction technique with a more controlled bimanual interaction (according to Guiard's model) can be proposed (see Figure 6).

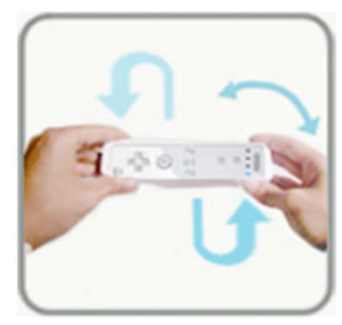

Fig. 6. Bimanual interaction technique. The bold blue arrows indicate user motion while accelerating and the thin blue arrows indicate user motion while navigating

Moreover, our objective was to extend the theoretical framework to a maze path. The study confirmed that the Steering Law established for the circular and straight paths in $3 \mathrm{D}$ environments is also valid for a maze path. The correlations in this 
experiment are slightly smaller than those obtained by Zhai and Woltjer [10]. These differences might be due to the inexperienced with new technologies population used in this study.

We can consider this study as a first step forward to better know the requirements for the input devices used in specific domain such as VR mental health technologies. We hope that this will motivate more researchers to carefully chose and design the input devices for their specific $\mathrm{MH}$ applications, which in turn will allow to create a more systematic typology of input devices for $\mathrm{MH}$ technologies.

Acknowledgements. This study was funded by Ministerio de Educación y Ciencia Spain, Project Game Teen (TIN2010-20187) and partially by projects Consolider-C (SEJ2006-14301/PSIC), "CIBER of Physiopathology of Obesity and Nutrition, an initiative of ISCIII" and Excellence Research Program PROMETEO (Generalitat Valenciana. Conselleria de Educación, 2008-157).

\section{References}

1. Frohlich, B., Hochstrate, J., Kulik, A., Huckauf, A.: On 3D Input Devices. IEEE Computer Graphics and Applications 26(2), 15-19 (2006)

2. Jin, W., et al.: Improving the visual realism of virtual surgery. In: Proc. Medicine Meets Virtual Reality, vol. 13, pp. 227-233 (2005)

3. Zudilova-Seinstra, E.V., de Koning, P.J.H., Suinesiaputra, A., et al.: Evaluation of 2D and 3D glove input applied to medical image analysis. International Journal of HumanComputer Studies 68(6), 355-369 (2010)

4. Botella, C., Quero, S., Baños, R.M., et al.: Virtual Reality and Psychotherapy. In: Riva, G., Botella, C., Légeron, P., Oplate, G. (eds.) Internet and Virtual Reality as Assessment and Rehabilitation Tools for Clinical Psychology and Neuroscience, IOS Press, Amsterdam (2004)

5. Zhai, S.: User Performance in Relation to 3D Input Device Design. Computer Graphics 32(4), 50-54 (1998)

6. Bowman, D.A., Kruijff, E., LaVoila Jr., J.J., Poupyrev, I.: 3D User Interfaces, Theory and practice. Addison-Wesley, Reading (2005)

7. Doherty, G., Coyle, D., Matthews, M.: Design and evaluation guidelines for mental health technologies. Interacting with Computers 22(4), 243-252 (2010)

8. Coyle, D., Doherty, G., Sharry, J.: PlayWrite: End-User Adaptable Games to Support Adolescent Mental Health. In: Proc. CHI (2010)

9. Accot, J., Zhai, S.: Beyond Fitts' Law: Models for Trajectory-Based HCI Tasks. In: Proceedings CHI, pp. 295-302 (1997)

10. Zhai, S., Woltjer, R.: Human Movement Performance in relation to path Constraint-The Law of Steering in Locomotion. In: Proc. IEEE Virtual Reality Conference (2003)

11. Lee, J.: Hacking the Nintendo WiiRemote. IEEE Pervasive Computing 7(7), 39-45 (2008)

12. Shernoff, D., Csikszentmihalyi, M., Schneider, B., Schernoff, E.: Student engagement in high school classrooms from the perspective of flow theory. Sch. Psy. Quar. 2, 158-176 (2003) 